\title{
Survival analysis of diagnostic assays in Plasmodium falciparum malaria
}

\author{
Melissa Phuong ${ }^{1}$, Rachel Lau², Filip Ralevski ${ }^{2}$ and Andrea K. Boggild ${ }^{2,3,4^{*}}$
}

\begin{abstract}
Background: Rapid diagnostic tests (RDT) and real-time PCR ( $\mathrm{PPCR}$ ) assays are sensitive for diagnosing malaria, but because they detect antigen and DNA, respectively, positivity may not reflect active infection. Performance characteristics of RDT and QPCR in Plasmodium falciparum positive specimens were evaluated over time to elucidate duration of positivity following conversion to microscopy negative.
\end{abstract}

Methods: Specimens from patients with at least one specimen that was positive for P. falciparum by microscopy, and at least one specimen that was negative for $P$. falciparum within a 1-month period were identified. Survival distributions of the diagnostic tests over time were compared. Performance characteristics for each test were calculated.

Results: Ninety specimens were included, with 48 initially positive for P. falciparum, and 42 subsequently negative. Of 42 specimens that converted to microscopy-negative following an initial positive, 26 (61.9\%) and 41 (97.6\%) were positive by qPCR and RDT, respectively. Survival curves of microscopy versus qPCR, as well as microscopy vs RDT differed significantly ( $p=0.0002$ and $p<0.0001$, respectively). Compared to microscopy, sensitivity of qPCR was $100.0 \%$ (95\% Cl 90.8-100.0\%), and that of RDT was $100.0 \%$ (95\% Cl 90.8-100.0\%).

Conclusions: Due to slow clearance of circulating antigen and DNA from bloodstream, RDT and qPCR have low positive predictive value for clinically relevant asexual parasitaemia in post-treatment specimens. Thus, microscopy remains the only available malaria diagnostic that can reliably distinguish true asexual parasitaemia from prolonged clearance of antigen and nucleic acid in a convalescing patient.

Keywords: Plasmodium falciparum, Malaria diagnosis, PCR, Rapid antigen detection test

\section{Background}

Three common methods are used to diagnose malaria: microscopic examination of Giemsa-stained thick and thin blood smears; rapid diagnostic tests (RDTs); and polymerase chain reaction (PCR) or other nucleic-acid based assays $[1,2]$. These tests have varying degrees of sensitivity and specificity for different Plasmodium species [2, 3], however, they are highly sensitive for detecting Plasmodium falciparum, as species-specific morphology, histidine-rich protein 2 (HRP-2), and species-specific $18 \mathrm{~S}$ rRNA gene can all be detected or amplified $[1,4]$.

\footnotetext{
*Correspondence: andrea.boggild@utoronto.ca

${ }^{3}$ Tropical Disease Unit, Division of Infectious Diseases, UHN-Toronto General Hospital, 200 Elizabeth Street, 13EN-218, Toronto, ON M5G 2C4 Canada

Full list of author information is available at the end of the article
}

Microscopy remains the gold standard diagnostic test for malaria due to its sensitivity and specificity in expert microscopist hands, although considerable expertise is required, and results are operator dependent $[2,4]$. RDTs are routinely implemented due to their relative simplicity and rapid turnaround time, enabling them to function as a point-of-care diagnostic. The specificity of RDTs can be low, particularly in the setting of persistent antigenaemia post-treatment of $P$. falciparum infection, as well as non-falciparum species [2, 5-7]. More recently, real-time polymerase chain reaction (qPCR) has become increasingly implemented, although its availability is limited to well-resourced reference facilities. qPCR assays have high sensitivity and specificity, and they may be used to confirm ambiguous microscopic results as well as identify the presence of mixed infections [1]. Given that RDTs and GPCR detect parasite antigen and DNA, respectively, 
specificity can be compromised if clearance of these parasite components from the bloodstream of the patient is slow $[5,7]$.

The aim of this study was to examine the length of time that $\mathrm{qPCR}$ and RDT results remain positive in specimens from patients recovering from recent $P$. falciparum infection. The natural history of HRP-2 and 18S DNA clearance relative to microscopy is herein described in a series of malaria-positive specimens submitted to our laboratory.

\section{Methods}

\section{Specimens}

Surplus and anonymized malaria-positive specimens entered into the malaria biobank were identified and included in the analysis if: multiple specimens from a single individual were entered with not more than 1-month between the first and second specimen; isolated $P$. falciparum malaria was confirmed microscopically for the first specimen, with conversion to microscopic negativity or declining parasitaemia on at least one subsequent specimen; complete RDT (BinaxNOW Malaria kit, Alere, $\mathrm{ME}$ ) and Giemsa-stained thick and thin film microscopy results within the biobank database; and submission to the laboratory between October 2008 and April 2014.

\section{DNA extraction}

DNA was extracted from banked whole blood specimens using DNA Mini Kit Blood or Body Fluid Spin Protocol (Qiagen, Germantown, MD, USA). For each specimen, $200 \mu \mathrm{L}$ of frozen whole blood was thawed from $-80^{\circ} \mathrm{C}$ and DNA was eluted with $60 \mu \mathrm{L} \mathrm{AE}$ buffer. DNA was then stored at $-20^{\circ} \mathrm{C}$ prior to amplification.

\section{Qualitative real-time PCR}

All specimens underwent the following qPCR assays: human beta-2-microglobulin (B2MG) extraction control, Plasmodium malariae/Plasmodium ovale speciesspecific duplex and P. falciparum/Plasmodium vivax species-specific duplex, as previously described [8]. Plasmodium falciparum/P. vivax species-specific duplex qPCR assay was conducted to confirm the isolated presence of $P$. falciparum and quantify copy number of the $18 \mathrm{~S}$ rRNA gene. Plasmodium malariae/P. ovale speciesspecific duplex qPCR was conducted to exclude initial mixed infection as well. All qPCR assays were run using ABI 7900HT Real-time PCR System using the following standard conditions: $50{ }^{\circ} \mathrm{C}$ for $2 \mathrm{~min}, 95^{\circ} \mathrm{C}$ for $10 \mathrm{~min}$, $95^{\circ} \mathrm{C}$ for $15 \mathrm{~s}$, and $60^{\circ} \mathrm{C}$ for $1 \mathrm{~min}$ ( 45 cycles), as previously described [8]. For each $25-\mu \mathrm{L}$ reaction, $12.5 \mu \mathrm{L}$ of TaqMan universal PCR master mix (Life Technologies),
$7.5 \mu \mathrm{L}$ of primer and probe mixes with concentrations previously reported $[1,2,8]$ and $5 \mu \mathrm{L}$ of DNA were used.

All qPCR amplification curves were analysed using a manual Ct threshold of 0.02 and an automatic baseline. Positive results were identified if the $\mathrm{Ct}$ value was $<40$ for the B2MG and $P$. falciparum/P. vivax species-specific duplex assays or $<38$ for the $P$. malariae/P. ovale speciesspecific duplex assay, as previously described [8].

\section{Quantitative real-time PCR}

In order to quantify gene copy number, serial dilutions of a P. falciparum $18 \mathrm{~S}$ rRNA DNA clone (MRA-177 ATCC $^{\circledR}$ 83, Virginia) that ranged from 9 to 91 million copies/ qPCR reaction were prepared and were included in each run of the $P$. falciparum/P. vivax species-specific duplex qPCR assay. The logarithm of gene copy number was plotted against $\mathrm{Ct}$ values for each concentration of the clone. A linear regression was conducted from this graph, and the equation was used to calculate gene copy number for each banked specimen.

\section{Statistical analysis}

The number of days between each specimen in a group of specimens for a single case were calculated. KaplanMeier survival curves were constructed, where an event was defined as either negative microscopy, qPCR, or RDT result. The alpha level $(\alpha)$ was predetermined to be 0.05 for the log-rank test of all three diagnostic procedures, and a Bonferroni correction was applied for post hoc analysis among the three diagnostic tests.

To evaluate test performance characteristics for subsequent samples received after the initial specimen, two sets of calculations were conducted: one using microscopy as the reference standard, and one using qPCR as the reference standard. For each scenario, sensitivity, specificity, positive predictive value (PPV), and negative predictive value (NPV) were calculated. Analysis was conducted using IBM SPSS Statistics (IBM, New York, USA) and graphs were prepared using GraphPad Prism 5 (GraphPad Software, La Jolla, CA, USA).

\section{Results}

In total, 90 specimens from 24 individuals entered into the malaria biobank were analysed.

\section{Microscopy, rapid diagnostic test, and real-time PCR comparison}

Twenty-four initial specimens and 66 subsequent specimens were received from 24 patients, where 48 initial specimens were positive for $P$. falciparum by microscopy, with 42 subsequently collected specimens converting to 
microscopy negative. Out of 90 specimens, 89 (98.9\%) were positive for HRP-2 by RDT, thus, 41 microscopynegative specimens (97.6\%) were positive by RDT. Seventy-four specimens (82.2\%) were positive by qPCR, thus 26 microscopy negative specimens $(61.9 \%)$ were positive by qPCR.

Time between the last $P$. falciparum-positive microscopic result, positive qPCR, and RDT is summarized in Fig. 1. qPCR and RDT remained positive up to 19 and 63 days following microscopic conversion to negativity, respectively. In two cases, multiple specimens submitted over a period of 2 months, and time between the last $P$. falciparum-positive microscopic result, positive qPCR, and RDT for these two cases with multiple specimens is summarized in Fig. 2.

The three survival distributions (Fig. 3) were found to be significantly different by log-rank test $\left[\chi^{2}(2)=39.62\right.$, $\mathrm{p}<0.0001]$. Specifically, survival distributions of microscopy and qPCR, as well as microscopy and RDT were found to differ significantly $\left[\chi^{2}(1)=14.11, p=0.0002\right.$ and $\chi^{2}(1)=31.79, \mathrm{p}<0.0001$, respectively].
Performance characteristics of qPCR and RDT compared to microscopy for samples received after the initial specimen are summarized in Table 1 . Sensitivity and specificity of qPCR were $100.0 \%$ (95 \% CI 82.8-100.0 \%), and $38.1 \%$ (95\% CI 24.0-54.3\%), respectively. PPV for qPCR was $48.0 \%$ (95\% CI 33.9-62.4 \%) and NPV was 100.0 \% (95 \% CI 75.9-100.0 \%). Compared to microscopy, sensitivity of RDT for asexual parasitaemia was $100.0 \%$ (95 \% CI 82.8-100.0 \%) and specificity was $2.4 \%$ (95\% CI 0.1-14.1\%). PPV and NPV for RDT were $36.9 \%$ (95\% CI 25.6-49.8 \%) and $100.0 \%$ (95 \% CI 5.5-100.0\%) compared to microscopy, respectively.

Performance characteristics of microscopy and RDT compared to qPCR are summarized in Table 2. Sensitivity and specificity of microscopy were $75.8 \%$ (95\% CI 63.4-85.1 \%) and $100.0 \%$ (95 \% CI 75.9-100.0\%), compared to qPCR, respectively. PPV was $100.0 \%$ (95\% CI $82.8-100.0 \%$ ) and NPV was $38.1 \%$ (95 \% CI $24.0-$ $54.3 \%)$. Compared to qPCR, sensitivity of RDT was $100.0 \%$ (95 \% CI 91.1-100.0 \%) and specificity was $6.3 \%$ (95\% CI 0.3-32.3\%). PPV and NPV for RDT were $98.5 \%$

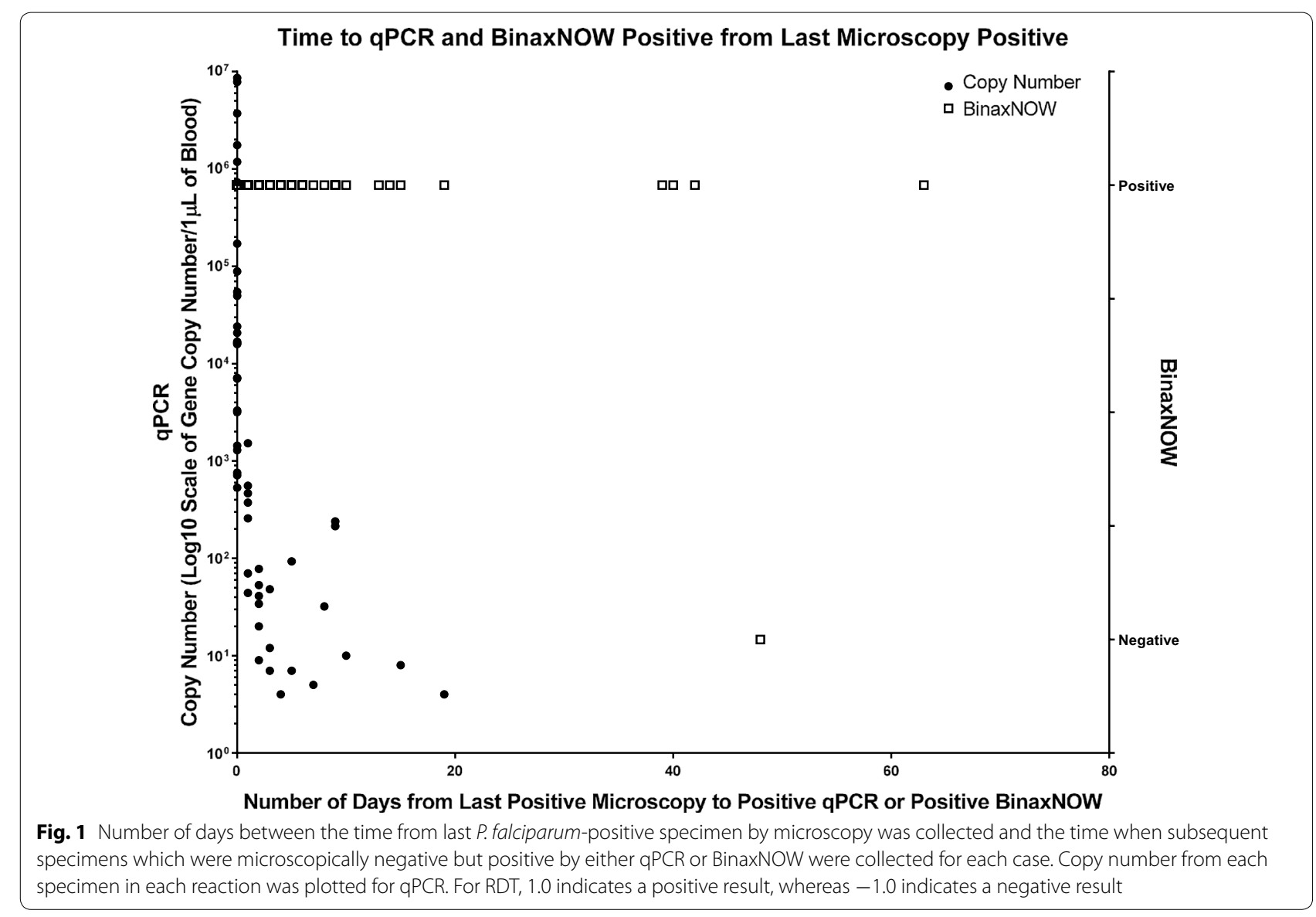




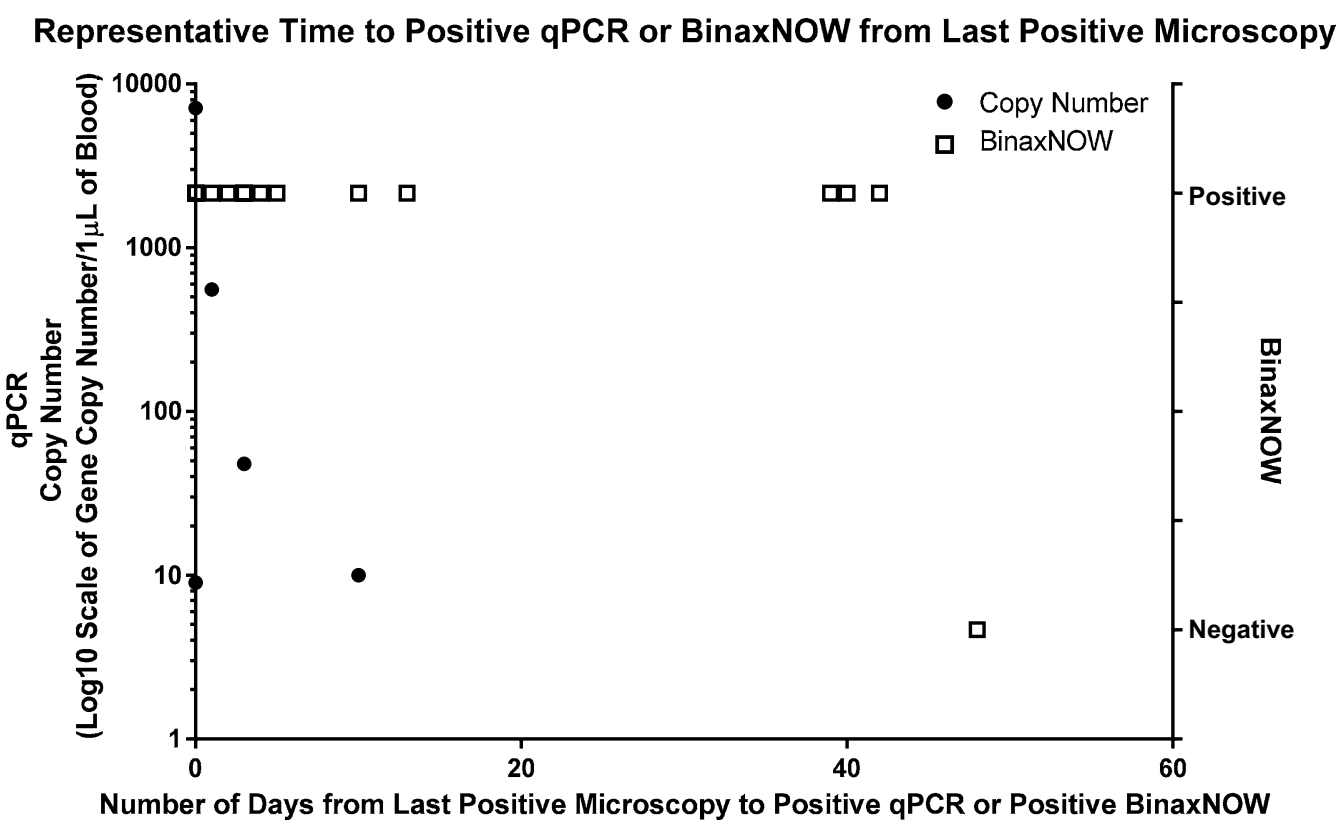

Fig. 2 Positivity of qPCR and RDT results following initial confirmation of P. falciparum-negative conversion by microscopy in two sets of specimens with frequent samples submitted over a 1-month period

(95\% CI 64.5-86.1 \%) and $100.0 \%$ (95 \% CI 5.5-100.0 \%) compared to $\mathrm{qPCR}$, respectively. RDT had a very low specificity and PPV for asexual P. falciparum parasitaemia, regardless of comparator.

\section{Discussion}

Of three main diagnostic platforms-microscopy, PCR, and RDT-microscopy is considered the gold standard $[9,10]$. Furthermore, microscopy is the only available diagnostic test for malaria that can reliably differentiate sexual from asexual parasitaemia. As PCR and RDT detect parasite components rather than the whole parasite itself, clearance from the bloodstream may be delayed $[2,7]$, and may yield false-positive results post-treatment. In addition, sexual stages of $P$. falciparum (gametocytes), which have no clinical relevance, may lead to positive RDT and/or qPCR, and this may be misinterpreted by clinicians as relapse or recrudescence of asexual parasitaemia. Recommendations to perform day-7 and day28 follow-up parasitaemia levels in falciparum malaria [11], were made prior to the widespread use of RDTs and qPCR assays in hematology and reference laboratories, and must be interpreted accordingly. Understanding the performance limitations of RDT and qPCR is imperative to correct interpretation of malaria diagnostic tests in clinical cases of malaria, particularly following appropriate administration of therapeutic anti-malarials. As laboratories are often not privy to ongoing clinical details in falciparum malaria, careful reporting of RDT and qPCR results must be undertaken, with sufficient exposition of limitations, particularly in the context of microscopic conversion to negativity.

Through survival analysis, we have documented the significant time difference in clearance of whole parasites, DNA, and HRP-2 following initial reporting of P. falciparum asexual parasitaemia. This study is limited in its ability to provide a robust estimate of clearance time for each test due to the small number of enrolled specimens and collection at different time points following the initial positive test. Furthermore, there is the potential for bias in the studied sample, as uncomplicated cases or patients who are semi-immune may be less likely to return for follow-up testing. Thus, more rapid parasite DNA or HRP-2 clearance may by under-represented in this study. Future studies with prospective enrolment of patients and daily follow-up of microscopic parasitaemia, RDT, and qPCR would address this limitation and provide a more precise estimate of clearance for each test.

\section{Conclusions}

Microscopy, qPCR, and RDT have variable performance characteristics and taken together, provide highly sensitive and specific detection of $P$. falciparum asexual parasitaemia. For follow-up post-treatment, however, microscopy still remains the only assay with adequate specificity for ongoing asexual parasitaemia and 


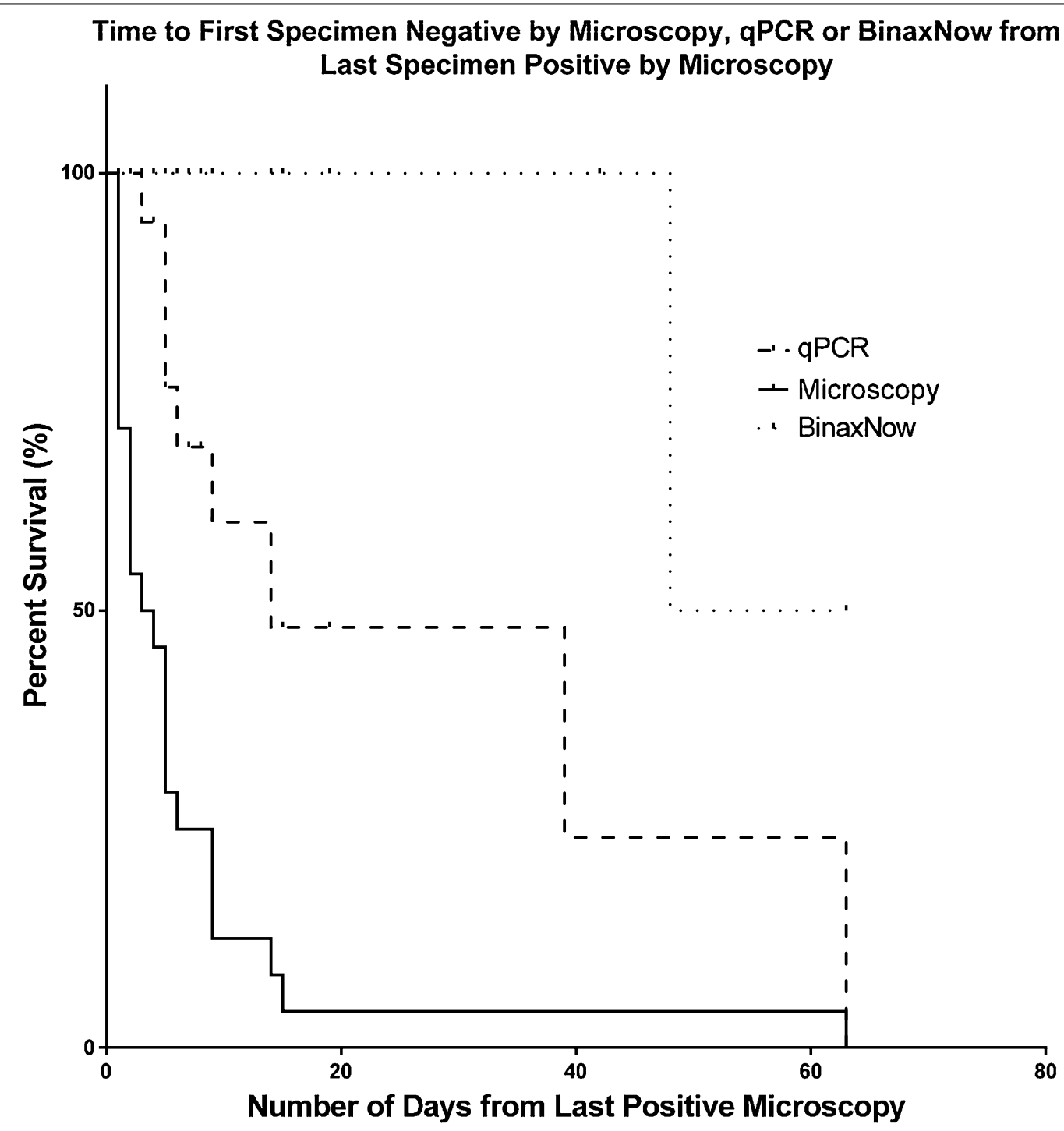

Fig. 3 Kaplan-Meier survival curves for all three diagnostic assays

Table 1 Performance characteristics of qPCR and BinaxNOW with microscopy as the reference standard for subsequent specimens

\begin{tabular}{|c|c|c|c|c|}
\hline & \multicolumn{4}{|c|}{ Reference standard: microscopy } \\
\hline & $\begin{array}{l}\text { True } \\
\text { positives }\end{array}$ & $\begin{array}{l}\text { True } \\
\text { negatives }\end{array}$ & $\begin{array}{l}\text { False } \\
\text { positives }\end{array}$ & $\begin{array}{l}\text { False } \\
\text { negatives }\end{array}$ \\
\hline $\mathrm{qPCR}$ & 24 & 16 & 26 & 0 \\
\hline BinaxNOW & 24 & 1 & 41 & 0 \\
\hline
\end{tabular}

with low relative risk of false positivity. Laboratories must be cognizant of the RDT and GPCR performance limitations that, if reported in isolation, may lead to
Table 2 Performance characteristics of microscopy and BinaxNOW with qPCR as the reference standard for subsequent specimens

\begin{tabular}{|c|c|c|c|c|}
\hline & \multicolumn{4}{|c|}{ Reference standard: qPCR } \\
\hline & $\begin{array}{l}\text { True } \\
\text { positives }\end{array}$ & $\begin{array}{l}\text { True } \\
\text { negatives }\end{array}$ & $\begin{array}{l}\text { False } \\
\text { positives }\end{array}$ & $\begin{array}{l}\text { False } \\
\text { negatives }\end{array}$ \\
\hline Microscopy & 24 & 16 & 0 & 26 \\
\hline BinaxNOW & 50 & 1 & 15 & 0 \\
\hline
\end{tabular}

misinterpretation of results and potential erroneous retreatment of malaria in a clinically-well patient free of asexual parasitaemia. 


\section{Authors' contributions}

$\mathrm{RL}$ and MP contributed to data collection, analysis, and interpretation, and to writing the manuscript. FR contributed to data collection and analysis, and to critical appraisal of the manuscript. AKB conceived the study, contributed to data analysis and interpretation, and to writing the manuscript. All authors critically reviewed the manuscript. All authors read and approved the final manuscript.

\section{Author details}

${ }^{1}$ Faculty of Health Sciences, McMaster University, Hamilton, Canada. ${ }^{2}$ Public Health Ontario Laboratories, Public Health Ontario, Toronto, Canada. ${ }^{3}$ Tropical Disease Unit, Division of Infectious Diseases, UHN-Toronto General Hospital, 200 Elizabeth Street, 13EN-218, Toronto, ON M5G 2C4, Canada. ${ }^{4}$ Department of Medicine, University of Toronto, Toronto, Canada.

\section{Acknowledgements}

We thank MR4 for providing us with Plasmodium small subunit ribosomal RNA clones contributed by Peter A. Zimmerman. This work was funded by the Public Health Ontario Laboratories (PHOL).

\section{Compliance with ethical guidelines}

\section{Competing interests}

The authors declare that they have no competing interests.

Received: 6 July 2015 Accepted: 3 September 2015

Published online: 17 September 2015

\section{References}

1. Khairnar K, Martin D, Lau R, Ralevski F, Pillai DR. Multiplex real-time quantitative $P C R$, microscopy and rapid diagnostic immuno-chromatographic tests for the detection of Plasmodium spp: performance, limit of detection analysis and quality assurance. Malar J. 2009;8:284.

2. Shokoples SE, Ndao M, Kowalewska-Grochowska K, Yanow SK. Multiplexed real-time PCR assay for discrimination of Plasmodium species with improved sensitivity for mixed infections. J Clin Microbiol. 2009;47:975-80.

3. Wilson ML. Laboratory diagnosis of malaria: conventional and rapid diagnostic methods. Arch Pathol Lab Med. 2013;137:805.

4. Obare P, Ogutu B, Adams M, Odera JS, Lilley K, Dosoo D, et al. Misclassification of Plasmodium infections by conventional microscopy and the impact of remedial training on the proficiency of laboratory technicians in species identification. Malar J. 2013;12:113.

5. Thompson CA, Boggild AK. Rapid diagnostic tests for imported malaria. CMAJ. 2014;186:E557.

6. Farcas GA, Zhong KJY, Lovegrove FE, Graham CM, Kain KC. Evaluation of the Binax NOW ICT test versus polymerase chain reaction and microscopy for the detection of malaria in returned travelers. Am J Trop Med Hyg. 2003;69:589-92.

7. Kyabayinze DJ, Tibenderana JK, Odong GW, Rwakimari JB, Counihan H. Operational accuracy and comparative persistent antigenicity of HRP2 rapid diagnostic tests for Plasmodium falciparum malaria in a hyperendemic region of Uganda. Malar J. 2008;7:221.

8. Phuong M, Lau R, Ralevski F, Boggild AK. Sequence-based optimization of a quantitative real-time PCR assay for the detection of Plasmodium ovale and P. malariae. J Clin Microbiol. 2014;52:1068-73.

9. Wongsrichanalai C, Barcus MJ, Muth S, Sutamihardja A, Wernsdorfer WH. A review of malaria diagnostic tools: microscopy and rapid diagnostic test (RDT). Am J Trop Med Hyg. 2007:77:119-27.

10. WHO. Guidelines for the treatment of malaria. 2nd ed. Geneva: World Health Organization; 2010

11. WHO. World malaria report. Geneva: World Health Organization; 2009.

\section{Submit your next manuscript to BioMed Central and take full advantage of:}

- Convenient online submission

- Thorough peer review

- No space constraints or color figure charges

- Immediate publication on acceptance

- Inclusion in PubMed, CAS, Scopus and Google Scholar

- Research which is freely available for redistribution

Submit your manuscript at 